\title{
Melanoma of the middle ear: initial presentation, Fluoro-2-deoxy-D-glucose positron emission tomography/computed tomography imaging and follow up
}

\author{
M W HUELLNER ${ }^{1}$, M COUSIN $^{2}$, T LINDER $^{2}$, J D SOYKA ${ }^{3}$, C BUSSMANN $^{4}$, L EBERLE $^{5}$, \\ K STROBEL ${ }^{1,3}$ \\ Departments of ${ }^{1}$ Nuclear Medicine and Radiology, ${ }^{2}$ Otorhinolaryngology, Head and Neck Surgery and \\ ${ }^{4}$ Pathology, Cantonal Hospital Lucerne, ${ }^{3}$ Department of Nuclear Medicine, University Hospital Zurich, and \\ ${ }^{5}$ ORL Center Brunnen, Hospitals of Schwyz and Einsiedeln, Switzerland
}

\begin{abstract}
Background: We present a rare case of primary mucosal melanoma of the middle ear imaged with 18F-fluoro-2-deoxy-Dglucose positron emission tomography/computed tomography (FDG-PET/CT).

Method: Clinical, radiological, intra-operative and histological findings are discussed.

Results: An 88-year-old woman presented with intermittent otorrhoea of the left ear for several months. Otoscopy revealed a livid protrusion of the tympanic membrane. Melanoma was not suspected initially, but was established on transmembranous biopsy. Pre-operative 18F-fluoro-2-deoxy-D-glucose positron emission tomography/computed tomography revealed a mass lesion in the left tympanic cavity with high fluoro-deoxyglucose uptake, as well as an ipsilateral intraparotid lymph node metastasis. The patient underwent surgical treatment. The diagnosis of melanoma was confirmed histologically.

Conclusion: In this rare case, clinical, radiological and surgical findings led to the diagnosis of a primary mucosal melanoma of the middle ear.
\end{abstract}

Key words: Melanoma, Mucosal; Middle Ear; PET/CT; FDG

\section{Introduction}

The vast majority ( 95 per cent) of malignant melanoma arises from melanocytes, the pigment-producing cells located mainly in the basal layer of the epidermis. However, this tumour can also arise from melanocytes residing in noncutaneous tissues, as is the case for mucosal melanoma. ${ }^{1}$

The treatment of mucosal melanoma is challenging, and exact pre-operative staging is crucial. 18F-fluoro-2-deoxyD-glucose positron emission tomography/computed tomography (FDG-PET/CT) enables evaluation of the primary tumour extent as well as the detection of lymph node and distant metastases, all in one imaging procedure. The successful use of this imaging procedure in cases of mucosal melanoma of the head and neck has been previously reported. ${ }^{2}$

We report the first case of a middle-ear melanoma imaged with FDG-PET/CT.

\section{Case report}

An 88-year-old woman presented with intermittent otorrhoea of the left ear for several months. Otoscopy revealed a livid protrusion of the tympanic membrane. No suspicious neck lymph nodes were palpable.
Audiometry showed severe combined hearing loss of the left ear.

The differential diagnosis at this stage was middle-ear adenoma, paraganglioma, tympanic meningioma, malignant tumour and infectious diseases such as tuberculosis.

A transmembranous biopsy was undertaken and the diagnosis of melanoma was confirmed histologically.

Computed tomography was performed to evaluate the local extent of the lesion. This showed a mass lesion within the left middle ear with obliteration of the mastoid cells and erosion of the tegmen and skull base. The auricular ossicles were intact and there was no infiltration of the inner ear. Fluoro2-deoxy-D-glucose positron emission tomography/computed tomography was performed for further staging. This demonstrated increased FDG uptake (maximum standardized uptake value $\left.\left(\mathrm{SUV}_{\max }\right)=5.1\right)$ of the mass lesion in the left tympanic cavity (Figure 1a to 1c). Additionally, there was focal FDG accumulation in the ipsilateral parotid gland $\left(\mathrm{SUV}_{\max }=\right.$ 9.1) (Figure 1d), suspicious for intraparotid lymph node metastasis. No distant metastases were visible on FDGPET/CT imaging.

The patient underwent subtotal petrosectomy and melanoma resection as well as lateral left-sided parotidectomy and a modified left-sided neck dissection. Intra-operatively, 


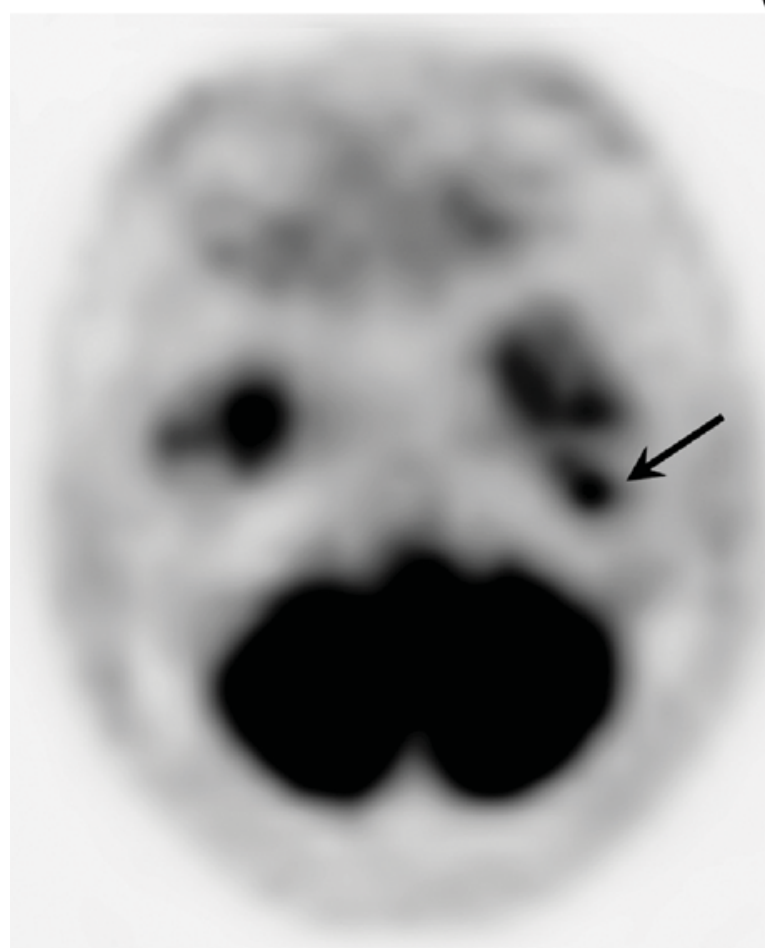

(a)

(b)

(c)
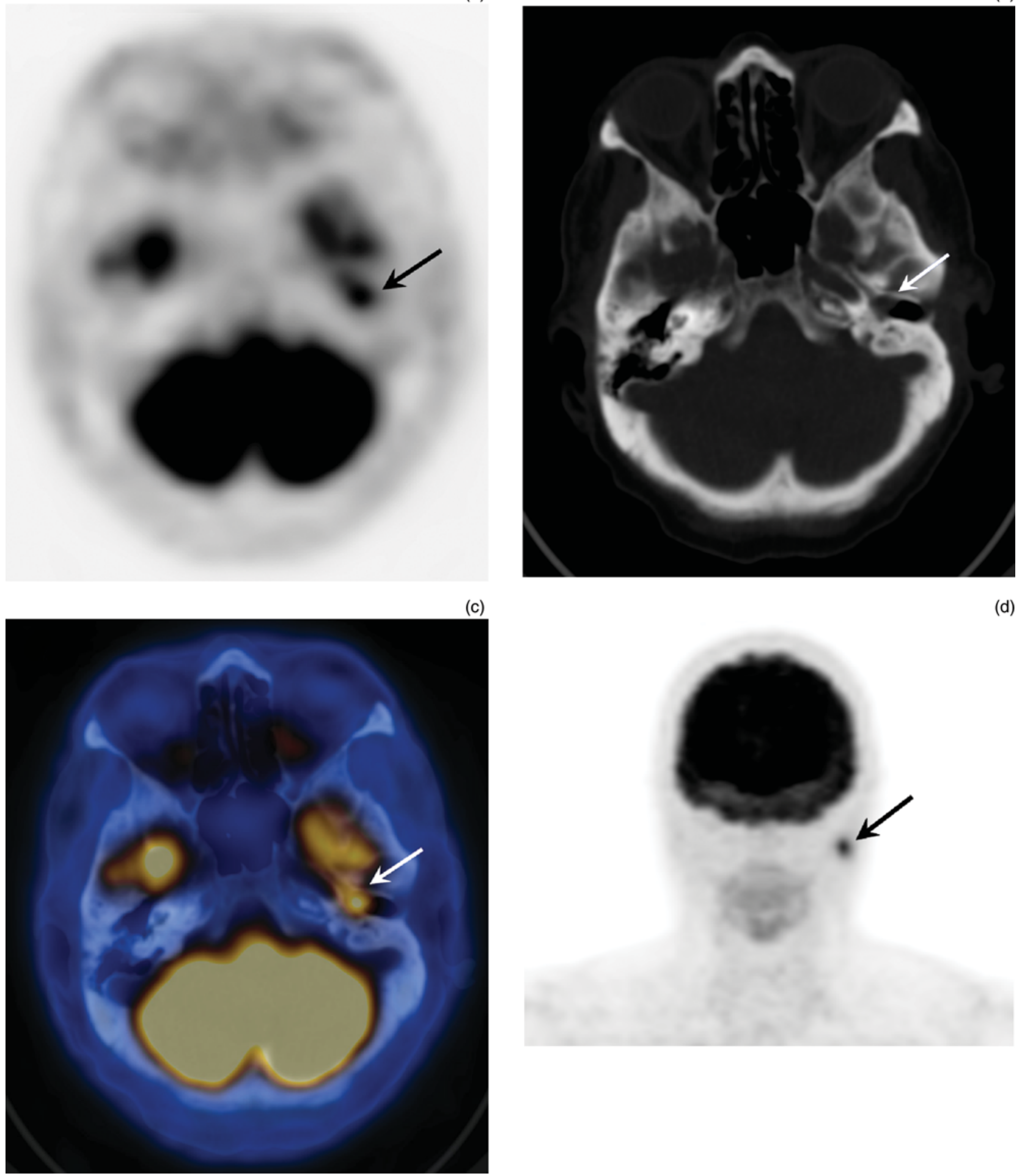

(d)

FIG. 1

(a)-(c) Axial Fluoro-2-deoxy-D-glucose positron emission tomography/computed tomography (FDG-PET/CT) scans showing FDG-active mass lesion in the left tympanic cavity (arrows). (d) Coronal FDG-PET/CT SCAN showing FDG accumulation in the ipsilateral parotid gland (arrow).

the lesion presented as a dark, brittle tumour at the tympanic orifice of the eustachian tube (Figure 2).

Immunohistological examination of the resected middleear tumour confirmed a mucosal melanoma by immunopathological positivity for S-100 (Figure 3a) and Melan-A
(Figure 3b). The lesion in the parotid gland was confirmed as a lymph node metastasis.

The patient recovered quickly from surgery.

Four months later, an enlarged, left-sided, level II cervical lymph node was palpated. Subsequent fine needle aspiration 


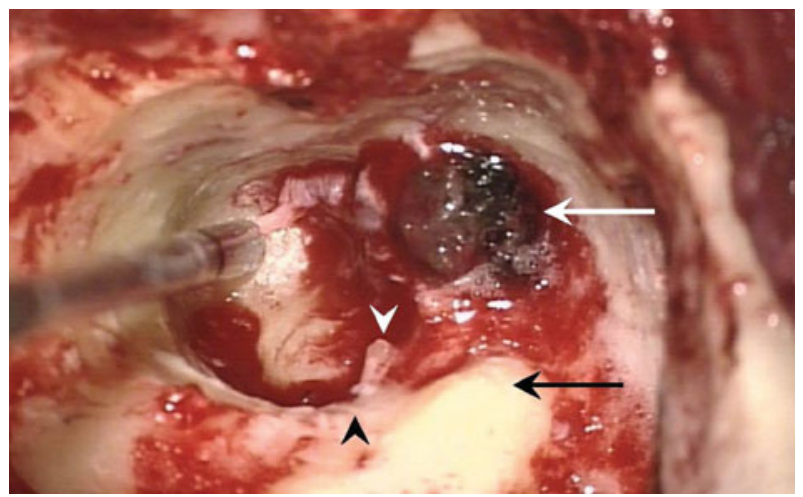

FIG. 2

Intra-operative view showing a brittle, black and purple tumour at the tympanic orifice of the Eustachian tube (white arrow). The stapes (white arrowhead), lateral semicircular canal (black arrow) and facial nerve (black arrowhead) are also visible.

(a)

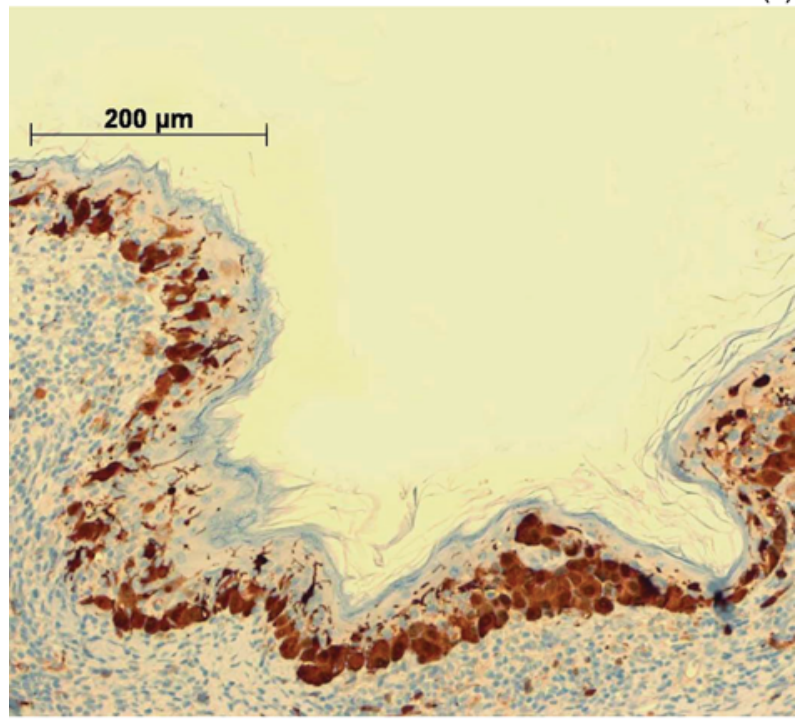

(b)

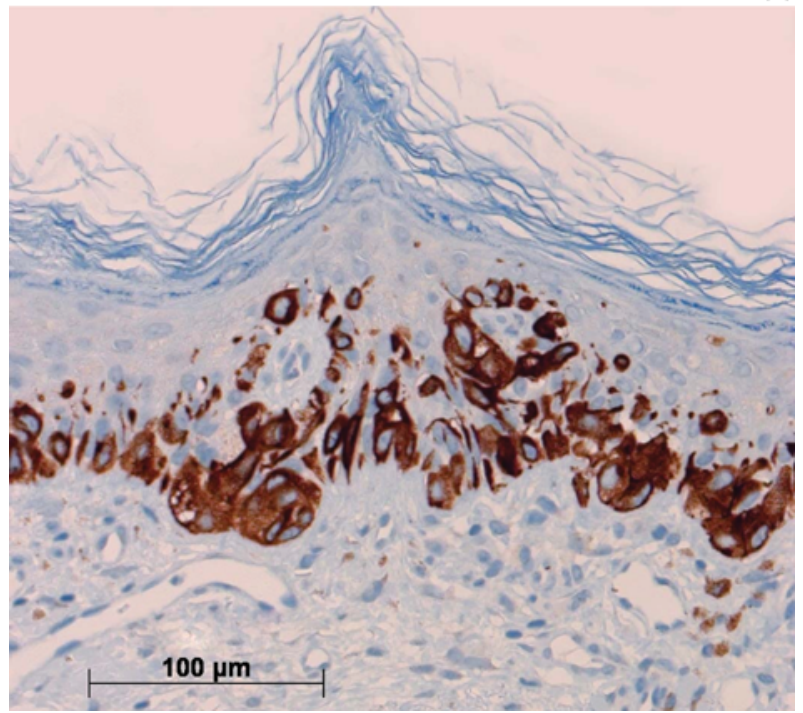

FIG. 3

Photomicrographs showing tympanic membrane with in situ malignant melanoma, with immunopathological positivity for (a) S-100 and (b) Melan-A. cytology revealed a melanoma metastasis. Neck dissection (level II and III) was performed, but revealed no evidence of additional lymph node metastases.

Six months after initial presentation, the patient developed multiple distant metastases in the liver and abdominal lymph nodes and died.

\section{Discussion}

A few case reports of malignant melanoma of the middle ear have previously been published. ${ }^{3-6}$ Approximately 1.3 per cent of all melanomas arise within the mucosal surfaces of the body, and 55 per cent of these occur in the head and neck. ${ }^{7}$

The treatment of mucosal melanomas is challenging. The only curative treatment is complete resection, but clear surgical margins are often difficult to obtain and this is considered to contribute to the poor prognosis. ${ }^{7,8}$ Some studies suggest that improved local control could be achieved by post-operative radiotherapy. ${ }^{8}$

Malignant melanoma can metastasise to any organ, and the location of metastases is often unpredictable. Metastasising melanoma is refractory to chemotherapy in most cases, although several clinical trials of vaccines and cytokines are in progress. ${ }^{9}$ Recent studies suggest a therapeutic option involving inhibiting signalling pathways in tumours with the v-Raf murine sarcoma viral oncogene homolog B1 gene (BRAF) mutation. ${ }^{10}$ If distant metastasis of melanoma occurs then the outcome is poor, with an average survival of only four to six months. ${ }^{1,11}$

Of the above-mentioned patients with middle-ear melanoma, only half survived longer than eight months after diagnosis.

Clinical evaluation and conventional imaging modalities generally underestimate the true extent of malignant melanoma. Autopsies of patients with known cutaneous malignant melanoma have revealed a higher prevalence of metastases than is generally clinically documented. ${ }^{12-14}$

- This paper reports a rare case of primary mucosal melanoma of the middle ear, imaged with fluorodeoxyglucose positron emission tomography/ computed tomography (FDG-PET/CT)

- In melanoma cases, pre-operative FDG-PET/CT is a valuable aid to surgical planning and oncological treatment

- The presented patient underwent subtotal petrosectomy and melanoma resection plus lateral left-sided parotidectomy and a modified left-sided neck dissection

Precise staging of malignant melanoma is essential for early detection of metastases and initiation of appropriate treatment. ${ }^{11,15}$ The majority of the available literature suggests that FDG-PET/CT is the most accurate imaging modality for the detection of metastases in melanoma patients, due to the high avidity of FDG for melanoma tumour cells. ${ }^{16-18}$

\section{Conclusion}

Malignant melanoma of the middle ear is a very rare entity. However, it should be considered in the differential diagnosis of mucosal tumours. It may mimic other conditions found in the middle ear, and may produce similar symptoms such as 
hearing loss and otorrhoea. Whenever melanoma is considered, the patient should undergo FDG-PET/CT to search for local and distant metastases, which if found may modify surgical and oncological treatment.

\section{References}

1 Markovic SN, Erickson LA, Rao RD, Weenig RH, Pockaj BA, Bardia A et al. Malignant melanoma in the 21 st century, part 1 epidemiology, risk factors, screening, prevention, and diagnosis Mayo Clin Proc 2007;82:364-80

2 Goerres GW, Stoeckli SJ, von Schulthess GK, Steinert HC FDG PET for mucosal malignant melanoma of the head and neck. Laryngoscope 2002;112:381-5

3 McKenna EL Jr, Holmes WF, Harwick R. Primary melanoma of the middle ear. Laryngoscope 1984;94:1459-60

4 Sherman IW, Swift AC, Haqqani MT. Primary mucosal malignant melanoma of the middle ear. J Laryngol Otol 1991;105: 1061-4

5 Curry JM, Kung BC, Willcox TO Jr. Malignant melanoma of the middle ear. Otolaryngol Head Neck Surg 2007;136:856-7

6 Uchida M, Matsunami T. Malignant amelanotic melanoma of the middle ear. Arch Otolaryngol Head Neck Surg 2001;127: 1126-8

7 Patel SG, Prasad ML, Escrig M, Singh B, Shaha AR, Kraus DH et al. Primary mucosal malignant melanoma of the head and neck. Head Neck 2002;24:247-57

8 Bradley PJ. Primary malignant mucosal melanoma of the head and neck. Curr Opin Otolaryngol Head Neck Surg 2006;14 $100-4$

9 Thompson JF, Scolyer RA, Kefford RF. Cutaneous melanoma. Lancet 2005;365:687-701

10 Dhomen N, Marais R. BRAF signaling and targeted therapies in melanoma. Hematol Oncol Clin North Am 2009;23:529-45, ix

11 Balch CM, Soong SJ, Atkins MB, Buzaid AC, Cascinelli N, Coit DG et al. An evidence-based staging system for cutaneous melanoma. CA Cancer J Clin 2004;54:131-49, 82-4

12 Amer MH, Al-Sarraf M, Vaitkevicius VK. Clinical presentation, natural history and prognostic factors in advanced malignant melanoma. Surg Gynecol Obstet 1979;149:687-92
13 Sampson JH, Carter JH Jr, Friedman AH, Seigler HF. Demographics, prognosis, and therapy in 702 patients with brain metastases from malignant melanoma. $J$ Neurosurg 1998;88:11-20

14 Budman DR, Camacho E, Wittes RE. The current causes of death in patients with malignant melanoma. Eur $J$ Cancer 1978;14:327-30

15 Balch CM, Buzaid AC, Soong SJ, Atkins MB, Cascinelli N, Coit DG et al. Final version of the American Joint Committee on Cancer staging system for cutaneous melanoma. J Clin Oncol 2001;19:3635-48

16 Friedman KP, Wahl RL. Clinical use of positron emission tomography in the management of cutaneous melanoma. Semin Nucl Med 2004;34:242-53

17 Reinhardt MJ, Joe AY, Jaeger U, Huber A, Matthies A, Bucerius $\mathrm{J}$ et al. Diagnostic performance of whole body dual modality $18 \mathrm{~F}-\mathrm{FDG}$ PET/CT imaging for $\mathrm{N}$ - and M-staging of malignant melanoma: experience with 250 consecutive patients. J Clin Oncol 2006;24:1178-87

18 Krug B, Crott R, Lonneux M, Baurain JF, Pirson AS, Vander Borght T. Role of PET in the initial staging of cutaneous malignant melanoma: systematic review. Radiology 2008;249: 836-44

Address for correspondence:

Dr Martin Huellner,

Department of Nuclear Medicine and Radiology,

Cantonal Hospital Lucerne,

CH-6000 Lucerne, Switzerland

Fax: +41412054689

E-mail: martin.huellner@ksl.ch

Dr M Huellner takes responsibility for the integrity of the content of the paper

Competing interests: None declared 